\title{
What are Pulsating Stars?
}

THose who are anxious that Europe should make the most of its technical facilities must find it satisfying that what may turn out to be one of the most important discoveries in astronomy in recent years was made on the east of the Atlantic. By now it is well known how the discovery of pulsars had to await the completion last July of the one-mile radio telescope at Cambridge, how the radio pulses were at first attributed to interference until parallax measurements indicated a source outside the solar system, and how an explanation in terms of vibrating neutron stars eventually seemed probable. Since then, the Mullard Radio Astronomy Observatory and Hoyle's Institute of Theoretical Astronomy at Cambridge have made much of the running, together with the Nuffield Radio Astronomy Laboratories at Jodrell Bank. That the first reports of optical pulsations from one of the pulsars should come from the United. States is hardly surprising, however; clouded skies make Europe a poor location for optical astronomy.

The detection by the Kitt Peak and Lick Observatories of optical pulsations from $C P$ 1919, reported at the New York conference on pulsars, came when astronomers were beginning to suspect that the pulsa. tions were only in the radio spectrum. This itself would have been difficult to explain, but the report that the period of the optical pulses is twice that of the radio pulses, and the possibility that optical pulses at other harmonics of the radio pulses exist, does not make the problem any easier. $C P 1919$ is the pulsar tentatively identified with an 18th magnitude blue star by Sir Martin Ryle and Miss Judy Bailey earlier this year, but the discovery of optical pulsations does not mean the identification is now certain. Although the Kitt Peak telescope was pointed at the blue star, the field of view of the electronic detection equipment connected to the instrument was such that light pulses from stars within $20^{\prime \prime}$ of arc of the blue star could be detected. This means that the Kitt Peak measurements can be explained if light from the blue star was varying by 4 per cent, or if light from a nearby fainter object was varying by a larger amount.

So far, theoretical interpretation of the pulsed radio signals has barely kept pace with the flow of new measurements. At first, an explanation in torms of the radial oscillation of compact dense stars seemed likely. The notion that the signals are coming from the long sought neutron stars was put forward chiefly because of the approximately 1 second interval between pulses; it seemed inconceivable that white dwarf stars-known to exist-could vibrate so rapidly. Any explanation has to account for two apparently incompatible features; the regularity of the period and the randomness of the pulse amplitude. In fact, the period is maintained so accurately that the problem of loss of energy by gravitational radiation is real, for oscillating neutron stars at least-white dwarfs do not seem to have this difficulty to the same extent. This led to explanations in terms of the gravitational lens effect in a binary system of neutron stars, for here there is a straw to cling to. There is controversy whether bodies in free-fall, as the two components of a binary are, emit gravitational energy according to general relativity, and thus pulsars became important as a testing ground for general relativity. The prediction of the binary theory that systematic differences between alternate pulses should exist was not borne out, however, and in spite of the diversity of the theories put forward since then, radial oscillation of compact stars may yet prove to be the solution. White dwarf stars, ruled out because their fundamental period of vibration seemed to be at least 8 seconds, may in fact vibrate with a period as low as $\mathbf{1} .5$ seconds, according to revised calculations.

How do the optical measurements from the United States fit in with this? As yet, the reports from Kitt Peak and Lick have not been assimilated by astronomers-many will no doubt want to see the measurements checked. The search will then be for a mechanism to generate light pulses at half the rate of the radio pulses. Possibly a rotating white dwarf can oscillate so as to emit pulses of radio energy in the direction of the Earth twice in each revolution, the light pulses coming from a flare-like disturbance on the star's surface triggered by shock waves from the oscillation. Whether or not an analysis along these lines can fit in with the observations now being made by radio observatories, the optical result suggests that rotation is somewhow involved.

\section{Opticians at Brighton}

\section{from a Correspondent}

The theme of the Southern Optical Congress, held at Brighton on May 21-23, "Future Developments", was interpreted by those attending as a renewed effort by the Association of Optical Practitioners and the British Optical Association to achieve full professional status for their members. The activity of these organizations to promote education and research was demonstrated by the congress programme. Miss J. Stone was awarded the BOA research medal for her investigation into the refractive properties of the growing eye. As a child ages, the eye enlarges, and the power of the cornea and lens alters to compensate.

Mr M. J. Gilkes lectured on retarded readers, giving a general survey of dyslexias as well as other social causes. Dr P. Jenner discussed unusual normal eye appearances, likely to be confused with pathology, and Dr L. J. Fish described the corneal and fundal changes associated with prolonged medication, some of which lead to opacification of the cornea or lens, and some to retinopathies. $\mathrm{Mr} \mathrm{A}$. J. Phillips described investigations into the effectiveness of commercially available "night driving" spectacles, many of which 